\title{
Anti-Proliferative and Apoptosis-Promoting Effect of microRNA-125b on Pancreatic Cancer by Targeting NEDD9 via PI3K/AKT Signaling
}

This article was published in the following Dove Press journal: Cancer Management and Research

\author{
Yuzheng Xue $\mathbb{D}^{1, *}$ \\ Yao Zhong ${ }^{1, *}$ \\ Tielong $\mathrm{Wu}^{\mathrm{I}}$ \\ Yingyue Sheng (D) \\ Yuanyuan Dai \\ Lingling $X u^{\prime}$ \\ Chuanqing $\mathrm{Bao}^{2}$
}

'Department of Gastroenterology, Affiliated Hospital of Jiangnan University, Wuxi 21404I, Jiangsu, People's Republic of China; ${ }^{2}$ Department of Gastrointestinal Surgery, Affiliated Hospital of Jiangnan University, Wuxi 21404I, Jiangsu, People's Republic of China

*These authors contributed equally to this work
Correspondence: Yuzheng Xue Department of Gastroenterology, Affiliated Hospital of Jiangnan University, No. 585, Xingyuan North Road, Wuxi 2/404I, Jiangsu, People's Republic of China

Email xueyz00I@I63.com
Purpose: The expression of microRNA-125b (miR-125b) is low in a variety of cancers, including gastric, lung, bladder, thyroid, and esophageal cancers. However, its specific mechanism in pancreatic ductal adenocarcinoma (PDAC) remains unclear. This study is aimed to explore the role of miR-125b in PDAC.

Methods: PDAC tissues and adjacent tissues were collected for miR-125b analysis by qRTPCR. Different PDAC cell lines were cultured for miR-125b detection by qRT-PCR, and CAPAN1 cells were selected for the downstream experiments. Cell proliferation was characterized by methyl thiazolyl tetrazolium (MTT) and 5-bromo-2-deoxyUridine (BrdU) staining. Flow cytometry was utilized for apoptosis and cell cycle changes. Cell invasion was determined by the Transwell assay and the dual-luciferase assay was utilized for validating the target gene. Western blotting was used to detect apoptosis related and PI3K/AKT signaling proteins.

Results: miR-125b was significantly down-regulated in human PDAC tissues and cell lines $(P<0.05)$. miR-125b inhibited the growth and invasion of CAPAN1 cells, facilitated apoptosis, and blocked the cell cycle at the G0/G1 phase. Furthermore, miR-125 directly targeted NEDD9. The high expression of NEDD9 impaired the anti-proliferative and antiapoptotic activity of miR-125b. miR-125b also inhibited apoptosis-related proteins and PI3K/AKT signaling pathways via NEDD9.

Conclusion: miR-125b decreased cell growth and invasion, and facilitated apoptosis in CAPAN1 cells through PI3K/AKT inhibition via targeting NEDD9.

Keywords: miR-125b, pancreatic ductal adenocarcinoma, apoptosis, NEDD9, PI3K/AKT

\section{Introduction}

Pancreatic ductal adenocarcinoma (PDAC) is a malignant tumor disease with poor prognosis. ${ }^{1,2}$ Due to the difficulty in initial diagnosis, high metastasis rate and drug resistance, the incidence and mortality associated with PDAC are extremely high and show an increasing trend. ${ }^{3}$ The 5-year survival rate of PDAC is less than $5 \%$. In China, the incidence of pancreatic cancer is fifth among the digestive system tumors, but the mortality rate is the second highest. ${ }^{4-6}$ Surgical resection is a better treatment for pancreatic cancer, but most patients miss the optimal timing window because they have no obvious symptoms in the early stage of the disease. ${ }^{7}$ Therefore, an in-depth study of the molecular mechanisms of pancreatic cancer may provide new theories and strategies for improving the therapeutic effect and prognosis of PDAC patients. 
MicroRNAs (miRNAs) are a class of about 20-nucleotide long endogenous non-coding RNAs present in eukaryotes that bind to mRNA and regulate gene expression. ${ }^{8}$ A recent study found the low expression of miR-125b in a variety of cancers, including gastric, lung, bladder, thyroid and esophageal cancers. ${ }^{9,10}$ Studies also reported that miR-125b was abnormally expressed in pancreatic cancer tissues and plasma. ${ }^{11,12}$ Jung and co-workers found that the different expression of miRNAs, including miR-100, miR-125b, miR-192, and miR-429, were detected in pancreatic cancer stem cells. ${ }^{13}$ Ottaviani et al reported that the potency of miR100 and miR-125b in PDAC was modulated by the TGF- $\beta$ pathway. ${ }^{14}$ However, the connection between miR-125b and its target gene, as well as the related signaling pathway, is still unknown.

Neural precursor cell expressed, developmentally down-regulated 9 (NEDD9), also known as HEF1, with metastasis-promoting activity in several types of cancers, ${ }^{15-17}$ is the predicted direct target of miR-125b.

In this study, our results showed that the expression of miR-125b was down-regulated in CAPAN1 cells, whereas its target NEDD9 was highly expressed. miR-125b inhibited growth and invasion and facilitated apoptosis in CAPAN1 cells. NEDD9 reversed the action of miR-125b.

\section{Materials and Methods}

\section{Tissue Specimen Collection}

Ten pairs of pancreatic cancer tissues and the corresponding adjacent tissues were collected. The adjacent tissues were $\geq 3 \mathrm{~cm}$ away from the focal point. The samples were obtained from pancreaticoduodenectomies conducted in the Department of Gastrointestinal Surgery at Affiliated Hospital of Jiangnan University, from November 2015 to June 2016. All specimens were stored in liquid nitrogen immediately after isolation. The study was approved by the Ethics Committee of Jiangnan University, and all subjects signed informed consent.

\section{Cell Culture}

The human pancreatic non-tumor cell line HPDE6 and human PDAC cell lines CAPAN1, SW1990, BXPC-3, and PANC-1 (purchased from Shanghai University Library of Chinese Academy of Sciences) were used in this study. The cells were cultured in $10 \%$ fetal bovine serum (Sigma, St. Louis, MO, USA) in complete medium DMEM (Gibco, Gaithersburg, MD, USA) (containing
$100 \mathrm{U} / \mathrm{mL}$ penicillin and $100 \mu \mathrm{g} / \mathrm{mL}$ streptomycin) at $37^{\circ}$ $\mathrm{C}$ and $5 \% \mathrm{CO}_{2}$ in saturated humidity conditions.

\section{RNA Isolation and qRT-PCR}

Total RNAs were isolated using the TRIzol ${ }^{\circledR}$ Plus RNA Purification Kit (Invitrogen, Carlsbad, CA, USA) based on the manufacturer's protocol. Total RNA was used to synthesize cDNA by reverse transcription using the SuperScript III First-Strand Synthesis Kit (Invitrogen, Carlsbad, CA, USA). Power SYBR Green PCR Master Mix (Applied Biosystems) was used for the real-time PCR analysis using an Applied Biosystems StepOnePlus ${ }^{\mathrm{TM}}$. Relative fold changes were calculated using the $2^{-\Delta \Delta \mathrm{Ct}}$ method and standard curves were produced. $\beta$-actin was used as an internal normalization control.

To evaluate the miR-125b levels, TaqMan MicroRNA assays (Applied Biosystems) were utilized based on the manufacturer's protocols. Small nuclear RNA U6 (Applied Biosystems) was as an endogenous control for the miRNA. The sequences of primers used were: BAX Forward: $5^{\prime}$ GGGTTGTCGCCCTTTTCTAC-3' and reverse: 5'-CTGG AGACAGGGACATCAGT-3'; Caspase3 Forward: 5'TGCTATTGTGAGGCGGTTGTAG-3' and reverse: 5'-G GCACACCCACCGAAAAC-3'; PI3K Forward: 5'-GTGT TTCATTGTCTAGTCCAACCTG-3' and reverse: 5'-GCAC TTGTAGGAATATGAAGCCTG-3'; AKT Forward: 5'-GG

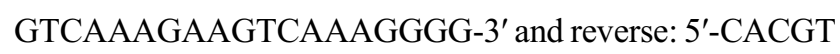
CGCTCATGGTGCC-3'; NEDD9 Forward: 5'-GAGCTGG ATGGATGACTACGA-3' and reverse: 5'-AGCTCTTTC TGTTGCCTCTCA-3'; U6 Forward: 5'-CTCGCTTCGGCA GCACA-3' and reverse: 5'-AACGCTTCACGAATTTGCG T-3'; has-miR125b Forward: 5'-GCAGTCCCTGAGACCCT $-3^{\prime}$ and reverse: $5^{\prime}$-CCAGTTTTTTTTTTTTTTTCACAAGT -3'; $\beta$-actin Forward: 5'-GATGAGATTGGCATGGCTTT-3' and reverse: 5'- GTCACCTTCACCGTTCCAGT-3'.

\section{Cell Transfection}

PDAC cells were plated in the logarithmic growth phase. After the cell density reached $80 \%$ fusion, miR- $125 \mathrm{~b}$ mimics, miR$125 \mathrm{~b}$ inhibitor, and their respective negative controls (miR-NC and siR-NC), purchased from GenePharma (Shanghai, China) were transferred into the well for $48 \mathrm{~h}$ according to Lipofectamine 2000 instructions. The NEDD9 expression plasmid and control vector pcDNA3.1 were obtained from GenePharma for the transfections and used according to the manufacturer's instructions. 


\section{MTT Assays}

The cells were reseeded into 96-well plates at a density of 8 x $10^{3} /$ well with different transfection plasmids. After 24,36 and $48 \mathrm{hrs}$ of incubation, respectively, $20 \mu \mathrm{L}$ of $5 \mathrm{mg} / \mathrm{mL}$ MTT was pipetted onto the plate. The cells were cultured at $37^{\circ} \mathrm{C}$ for another $4 \mathrm{hrs}$. Then, the medium was removed and $150 \mu \mathrm{L}$ of DMSO was added for 15 mins with shaking. The OD value was measured at $570 \mathrm{~nm}$ with a microplate reader. Three independent experiments were performed (three times each).

\section{Bromodeoxyuridine Incorporation Assay}

The bromodeoxyuridine (BrdU) incorporation assay was also utilized to evaluate cell proliferation. BrdU (Thermo Fisher Scientific, Waltham, MA, USA) was added at a concentration of $10 \mu \mathrm{M}$ for $48 \mathrm{hrs}$ and then was diluted with colchicine (Sigma-Aldrich) to $0.1 \mu \mathrm{M}$. The assays were conducted as previously described. The number of BrdU-positive cells and blue-stained nuclei were counted in five random fields under fluorescence microscopy.

\section{Apoptosis and Cell Cycle Assay}

Cells $\left(2 \times 10^{5}\right)$ were transfected for $48 \mathrm{hrs}$ and maintained in 6-well plate. Then the cells were washed with phosphate-buffered saline (PBS), fixed overnight with $70 \%$ cold ethanol, and stained with propidium iodide (PI). Finally, FACS Verse flow cytometry (BD, San Jose, CA, USA) was employed for the cell cycle analysis. The cells were stained with annexin V-FITC and PI for cell apoptosis analysis according to the manufacturer's instructions. The apoptotic rates were measured. All the assays were carried out independently triplets.

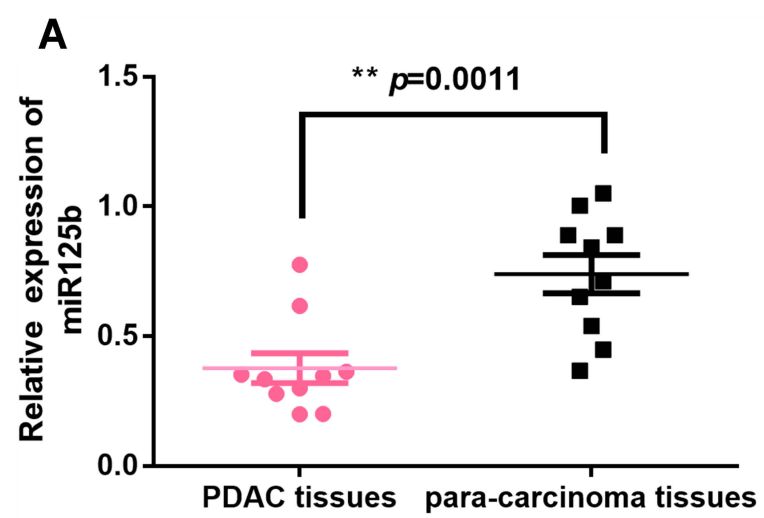

\section{Cell Invasion Analysis}

CAPAN1 cells were transfected for $48 \mathrm{~h}$, trypsinized and plated for the invasion assays. CAPAN1 cells $\left(2 \times 10^{5}\right)$ were incubated in the upper chamber in medium without serum in the Matrigel Invasion Chambers ( 8 microns) of 24-well plates (BD Bioscience, Franklin Lakes, NJ, USA). After $24 \mathrm{~h}$ of incubation, the cells on the other side of upper chambers were stained using $0.1 \%$ crystal violet for $30 \mathrm{~min}$ at $37^{\circ} \mathrm{C}$. The number of invading cells was counted in five random visual fields under a microscope.

\section{Western Blot Analysis}

Forty-eight hours after transfection, the CAPAN1 cells were lysed in RIPA buffer (Thermo Fisher Science, Waltham, MA, USA) and the cellular protein concentrations were determined with BCA kit (Beyotime, Nanjing, China). The proteins resolved on an SDS denatured polyacrylamide gel were transferred onto a nitrocellulose membrane (Millipore, Burlington, MA, USA). The membranes were incubated with diluted primary antibodies (anti-Bax, caspase 3, PI3K, AKT, NEDD 9 and $\beta$-actin, Abcam, USA) overnight at $4^{\circ} \mathrm{C}$. The membranes were washed and incubated with goat antimouse secondary antibodies $(1: 10,000)$ and visualized using an ECL kit (Millipore).

\section{Luciferase Activity Assay}

Wild-type (WT) or mutant (MUT) NEDD9-bound miR$125 \mathrm{~b}$ was inserted into a pmirloGLO dual luciferase small RNA targeting expression vector (Promega, Madison, WI, USA) to form a reporter vector (NEDD9/WT or NEDD9/ MUT). The cells were then transfected with NEDD9/WT

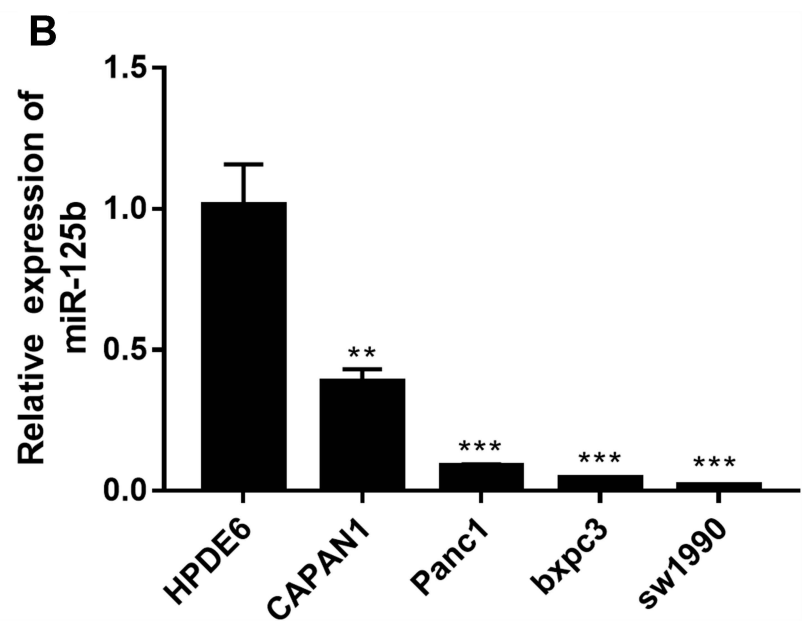

Figure I Low expression of miR-I25b in PDAC tissues and PDAC cell lines.

Notes: (A) miR-125b expression in paired PDAC tissues and adjacent tissues; (B) miR-I25b expression in PDAC cell lines by qRT-PCR analysis. $* * p<0.01$, $* * * p<0.001$ vs the respective controls. 
or NEDD9/MUT and/or miR-125b mimics or miR-NC for $48 \mathrm{hrs}$. The dual luciferase reporter assay system provided by Promega (Madison, WI, USA) was utilized to determine the luciferase activity.

\section{Statistical Analysis}

GraphpadPrism7.0 (GraphPad Software, USA) was applied to analyze the experimental data. All values are presented as the mean \pm SD for at least triplicate determinations. Differences between two groups were analyzed

with t-tests, whereas the statistical significance of differences between multiple groups was compared with ANOVA. $P<0.05$ was considered a significant difference.

\section{Results}

\section{miR-I25b Is Low-Expressed in PDAC Tissues and Cell Lines}

The expression level of miR-125b in pancreatic cancer tissues and adjacent tissues was firstly examined by qRTPCR. Figure 1A showed that the expression of miR-125b
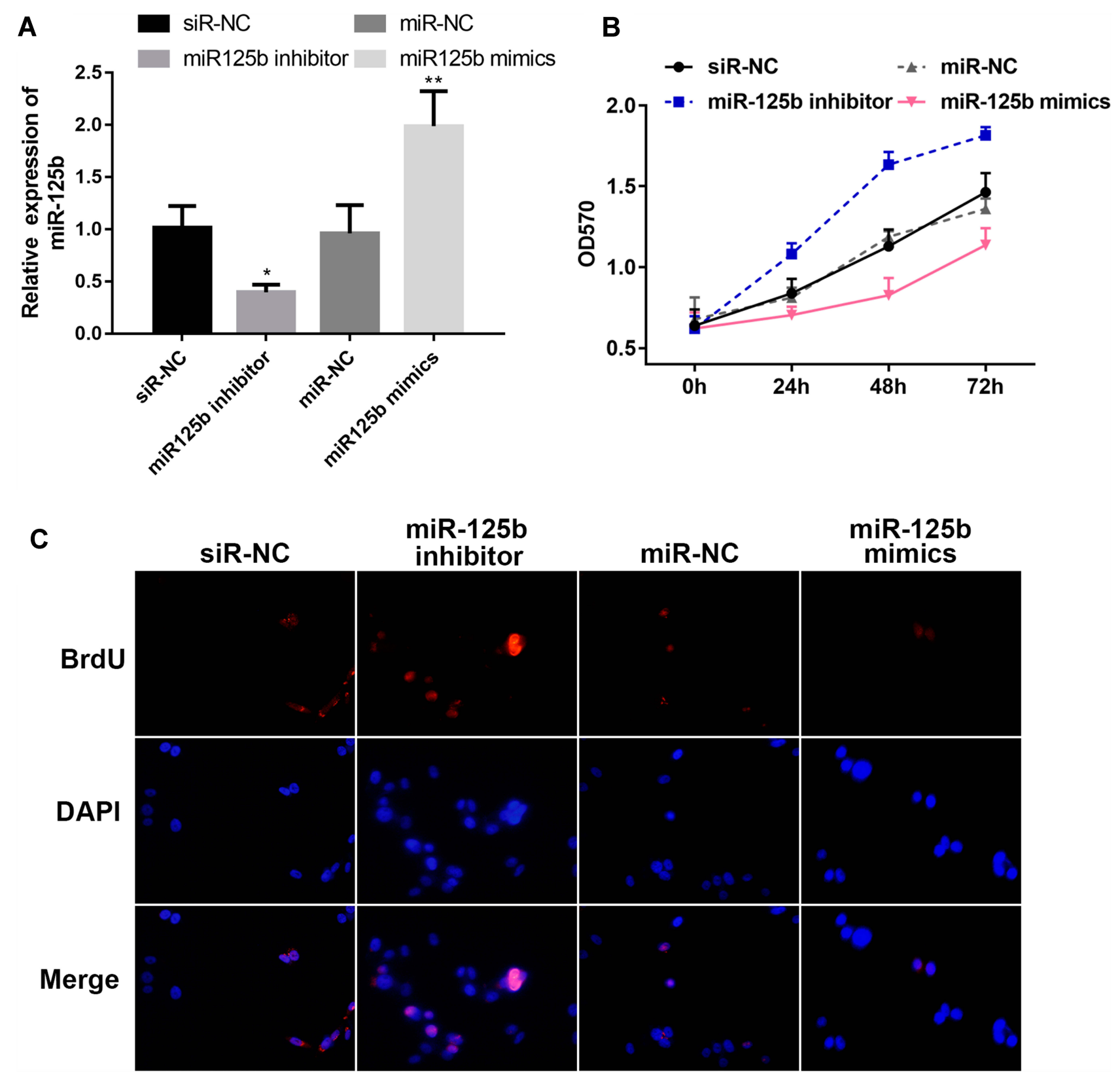

Figure 2 MiR-125b inhibits CAPANI cell proliferation.

Notes: (A) miR-125b levels in CAPANI cells with different plasmids; (B) proliferation rates of CAPANI cells determined by MTT transfected with different plasmids; (C) BrdU incorporation assay for cell proliferation. The values are presented as mean $\pm \operatorname{SD}(n=3), * p<0.05$, $* * p<0.01$ vs the respective controls. 
in PDAC tissues is significantly lower than that in the adjacent tissues. The expression of miR-125b was also determined in several PDAC cell lines (CAPAN1, SW1990, BXPC-3 and PANC-1), as well as in HPDE6 human normal pancreatic ductal epithelial cell line. The results in Figure 1B revealed that the low expression of miR-125b in PDAC cell lines compared to normal cells, indicating that miR-125b may play a role in the development and progression of PDAC. Because CAPAN1 cells showed the highest expression of miR-125b of the four PDAC cell lines, they were selected for subsequent experiments.

\section{miR-I25b Inhibits the Proliferation of CAPANI Cells}

In order to explore the function of miR-125b in CAPAN1 cells, the cells were transfected with different plasmids, divided into four groups: siR-NC, miR-125b inhibitor, miR-NC and miR-125b mimics according to the different treatments. The results of the qRT-PCR assay exhibited that the miR-125b level in the miR-125b mimics group in the CAPAN1 cells was higher than that of the miR-NC group, whereas that of the miR-125b inhibitor group was lower than that of the siR-NC group (Figure 2A).

For the proliferation activity, the results of the CAPAN1 cell growth curve obtained from the MTT assay were plotted. Transfection of CAPAN1 cells with miR-125b inhibitor increased the OD value compared to the siR-NC group. Conversely, transfection of miR-125b mimics reduced the rate of proliferation compared to the miR-NC group. Statistical analysis suggested a significant difference in the proliferative capacity between the miR-125b mimics group and the miR-125b inhibitor group (Figure 2B).

The results in Figure $2 \mathrm{C}$ show that the proliferation of cells in the miR-125b inhibitor group was enhanced compared with the siR-NC group, whereas the cell proliferation ability was significantly attenuated after transfection with miR-125b mimics. Thus, higher expression of miR$125 \mathrm{~b}$ inhibited CAPAN1 cell proliferation.

\section{miR-125b Induces Apoptosis, Regulates Cell Cycle and Inhibits Invasion in CAPANI Cells}

Followed with proliferation activity, the effect of miR$125 \mathrm{~b}$ on apoptosis, cell cycle and invasion were investigated. After $48 \mathrm{hrs}$ of transfection, the cells were collected and analyzed by flow cytometry. The number of apoptotic cells in the miR-125b inhibitor group was less than that in the siR-NC group, whereas it was significantly increased in the miR-125b mimics group (Figure 3A), suggesting that miR-125b promoted PDAC cell apoptosis.

To further investigate the growth inhibition observed following miR-125b expression, we compared the cellcycle profiles of cells with different transfections by flow cytometry. The results in Figure $3 \mathrm{~B}$ demonstrated that transfection with miR-125b led to an increasement in the number of cells in the G0/G1 phase.

The effect of miR-125b on the invasiveness of the CAPAN1 cells was also assessed with Transwell assays. Increased miR-125b significantly reduced the invasion potential of CAPAN1 cells, whereas low expression of miR-125b showed the opposite effect. These findings demonstrated that miR-125b suppressed CAPAN1 cells invasion (Figure 3C).

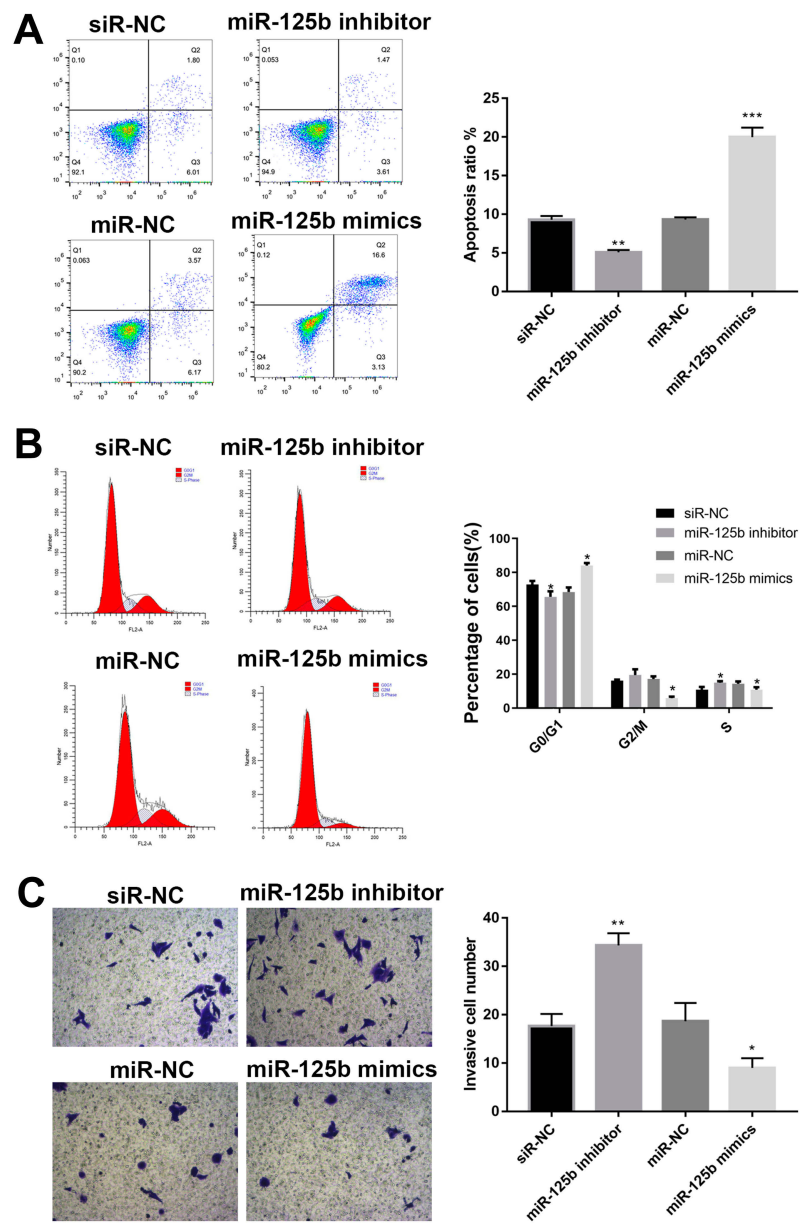

Figure 3 MiR-125b promotes apoptosis, regulates cell cycle and inhibits invasion in CAPANI cells.

Notes: (A) Apoptosis of cells with different plasmids by flow cytometry; (B) flow cytometric analysis of cell cycles; (C) representative images of cell invasion across the membrane. The mean values \pm SD $(n=3)$ are shown. ${ }^{*} p<0.05, *^{*} p<0.01$, $*_{* *}<<0.001$ vs the respective control. 


\section{miR-125b Regulates Apoptosis-Related} Proteins and PI3K/AKT Signaling Pathways in CAPANI Cells

Crucial proteins related to apoptosis and signaling pathways were validated by qRT-PCR and Western blots. The mRNA results in Figure 4A showed that the up-regulation of apoptosis-promoting genes (BAX and caspase 3 ) and the downregulation of PI3K and AKT mRNAs in the miR-125b mimics group compared with the miR-NC group, whereas the opposite results were seen in the miR-125b inhibitor group. At protein level, A similar trend was seen at the protein level (Figure 4B). These results suggest that miR125b played an anti-apoptotic role in CAPAN1 cells via regulating PI3K/AKT signaling pathway.

\section{miR-I 25b Directly Targets NEDD9}

To investigate the in-depth mechanisms underlying miR125b-mediated proliferation, apoptosis, cell cycle and invasion regulation, PicTar, Targetscan, miRWalk and the miRGen Target program were employed for predicting the target genes. The most promising candidate was NEDD9, which was predicted by most of the applied analysis. The binding site for miR- $125 \mathrm{~b}$ is present in the $3^{\prime} \mathrm{UTR}$ of the NEDD9 transcript, and it was demonstrated by the luciferase reporter assay in CAPAN1 cells (Figure 5A). The luciferase results in Figure 5B revealed that miR-125b significantly reduced the luciferase activity in the WT NEDD9 $(p<$ 0.05), whereas there was no change in the MUT-type. After the different transfections, the mRNA and protein levels of NEDD9 were determined. In the miR-125 inhibitor group, NEDD9 mRNA and protein levels were significantly upregulated. However, the miR-125 mimics group showed a decrease change (Figure 5C and D).

miR-125b Affects Proliferation, Invasion, Apoptosis-Related Proteins, and PI3K/AKT Signaling Pathways in CAPANI Cells via NEDD9

To verify whether miR-125b acts on CAPAN1 cells by targeting NEDD9, the cells were transfected and divided into three groups (miR-NC, miR-125b mimic and miR$125 \mathrm{~b}$ mimics + pcDNA3.1-NEDD9). In the miR-125b mimics
A
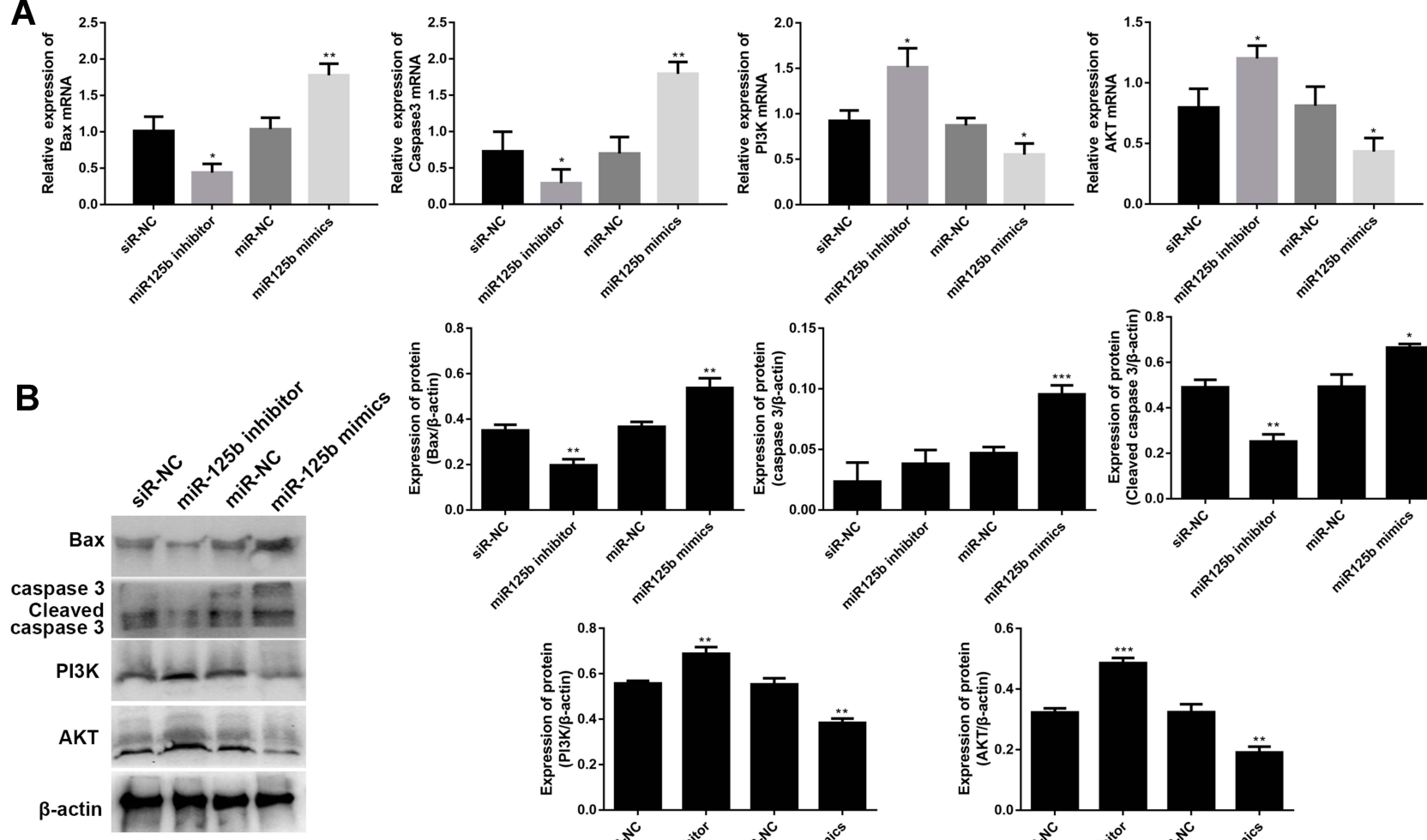
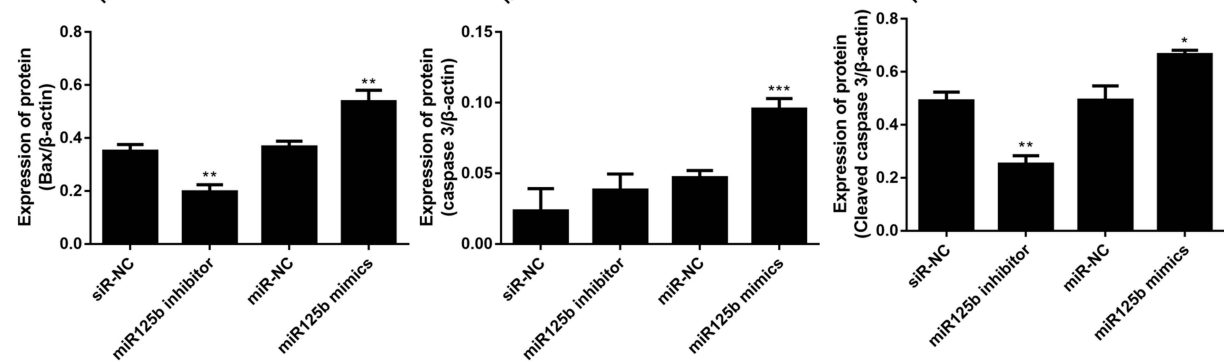
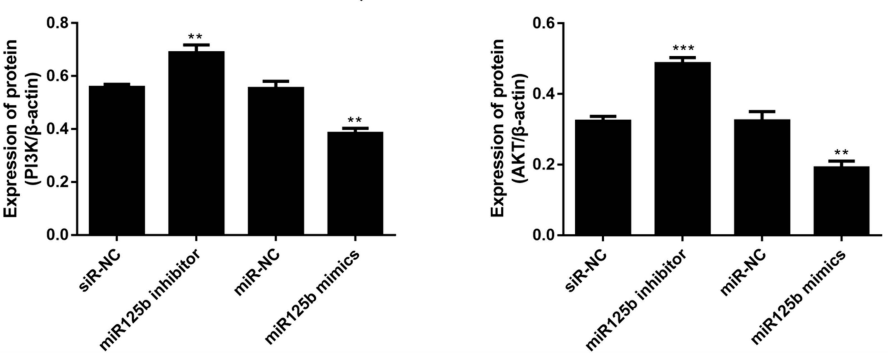

Figure 4 MiR-125b regulates apoptosis-related proteins and PI3K/AKT signaling pathways in CAPANI cells.

Notes: $(\mathbf{A})$ The mRNA levels of BAX, caspase 3, PI3K and AKT; $(\mathbf{B})$ the protein levels of BAX, caspase 3 , cleaved caspase 3 , PI3K and AKT. The mean values \pm SD $(n=3)$ are shown. ${ }^{*} p<0.05, * * p<0.01, * * * p<0.001$ vs the respective control. 

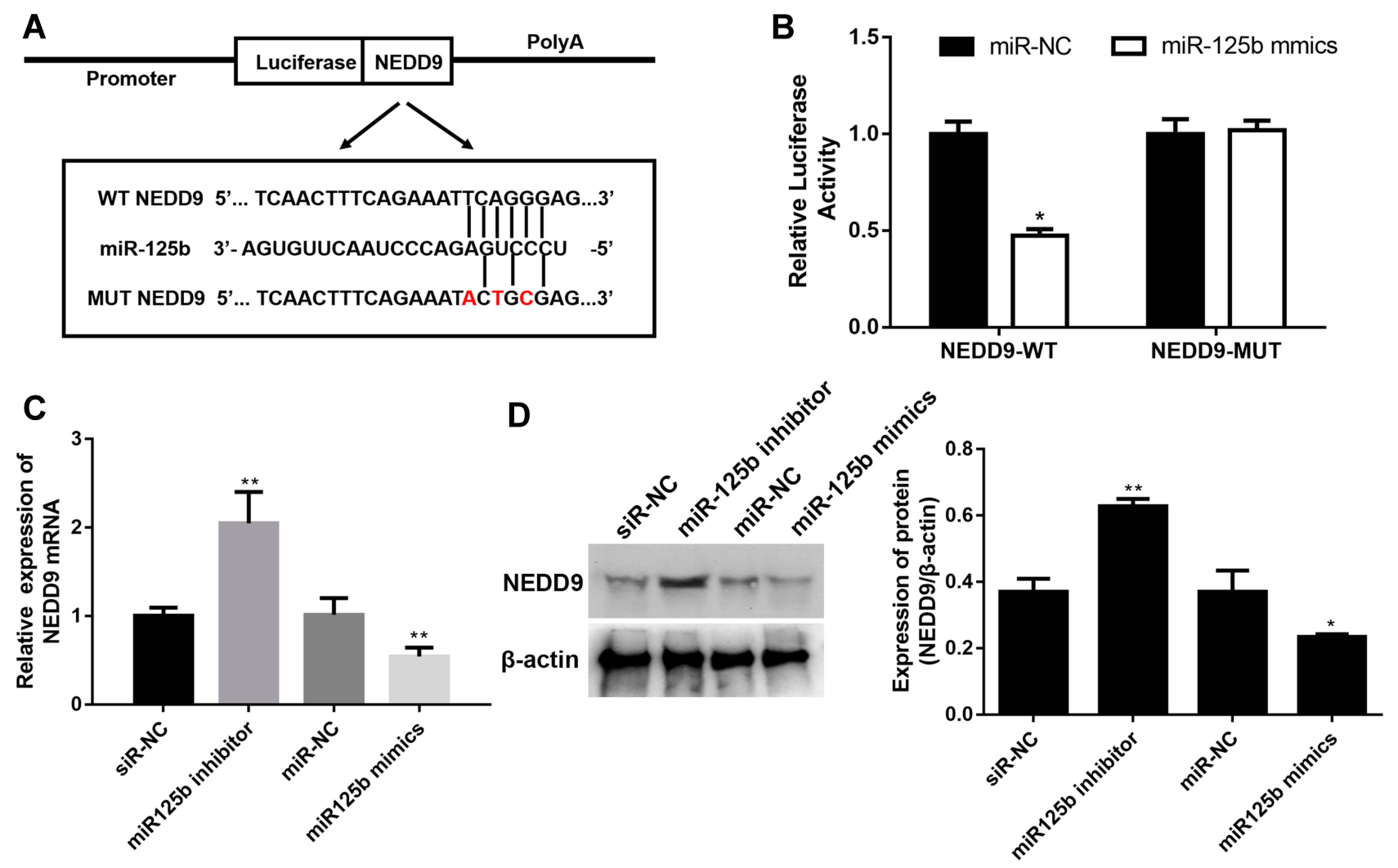

Figure 5 NEDD9 is a binding target of miR-125b.

Notes: (A) Binding region between miR-125b and NEDD9 and the luciferase reporter constructs containing the NEDD9/WT or NEDD9/MUT sequence. (B) Relative luciferase activity of miR-125b co-transfected into CAPANI cells. (C) Relative mRNA expression of NEDD9 in CAPANI cells with different transfections. (D) Western blot analysis and band intensity analysis of NEDD9 in CAPANI cells with different transfections. Data presented as mean \pm SD of three independent experiments. * $<0.05$, $*^{*} p<0.01$ vs the respective control.

group, NEDD9 was significantly reduced both at the mRNA and protein levels, whereas pcDNA3.1-NEDD9 reversed the reduction (Figure 6A and $\mathrm{B}$ ). Based on the MTT and BrdU assays, pcDNA3.1-NEDD9 significantly impaired the anti-proliferation potency of miR-125b mimics (Figure 6C and D). The transwell results indicated that the invasive ability of CAPAN1 cells in the miR-125b mimics group was restored due to overexpression of NEDD9 by pcDNA3.1-NEDD9 (Figure 6E).

Whether the apoptosis-related proteins and PI3K/AKT signaling pathway were altered by pcDNA3.1-NEDD9 were evaluated by qRT-PCR and Western blots. The mRNA level results in Figure 7A showed that the apoptosis promoting-genes (BAX and caspase 3) were significantly downregulated and PI3K and AKT mRNAs were significantly up-regulated after co-transfection with pcDNA3.1-NEDD9 compared to the miR-125b mimics. Figure 7B exhibited a similar trend at the protein level. These results suggest that miR-125b played an antiapoptotic role via targeting NEDD9.

\section{Discussion}

PDAC is one of the major causes of cancer-related deaths, so finding new molecular targets for its diagnosis, prognosis, and treatment may improve the clinical strategy and outcomes of the disease. ${ }^{18}$ Numerous studies have shown that miRNA expression was changed in pancreatic cancer, suggesting that miRNA dysregulation may exert functions in the development of pancreatic cancer. ${ }^{19}$ miRNAs have been shown to be abnormal in a variety of malignant tumors, and their expression levels have been associated with the diagnosis and prognosis of diverse types of cancers.

In this study, we transfected CAPAN1 cells with miR$125 \mathrm{~b}$ mimics and inhibitor to explore the effects of overexpression and knockdown of miR-125b on proliferation and apoptosis. The MTT assay indicated that the proliferative capacity of the miR-125b mimics group was remarkably inhibited compared with the miR-NC group, whereas that of the miR-125b inhibitor group was remarkably higher. Similarly, the BrdU experiment revealed that after 
A

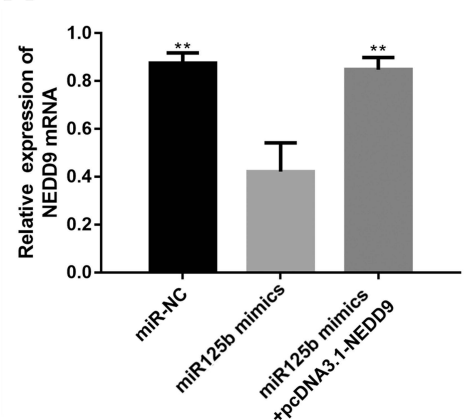

C

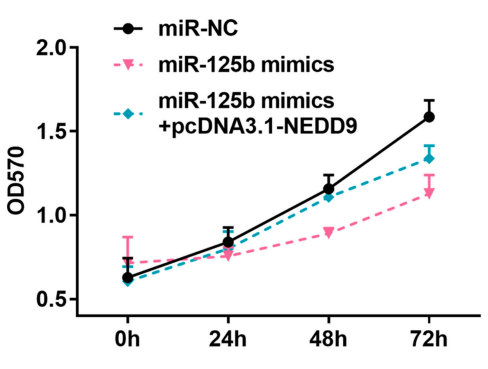

B

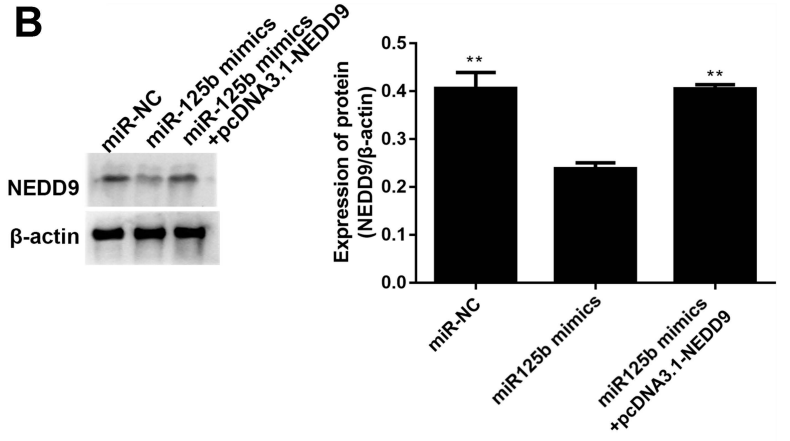

D

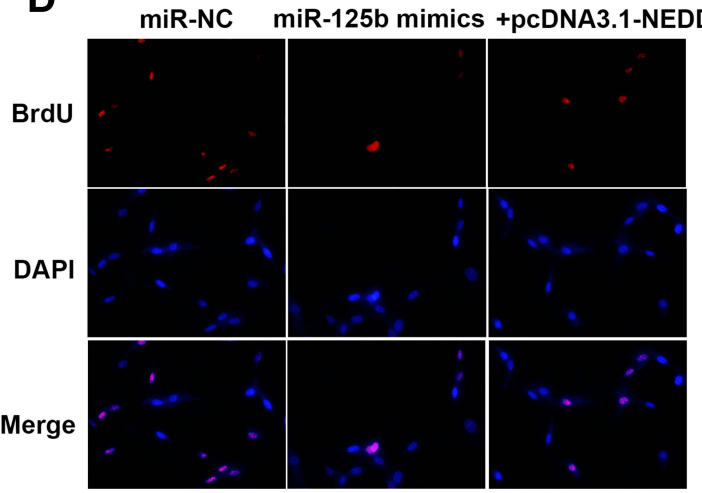

$\mathbf{E}$

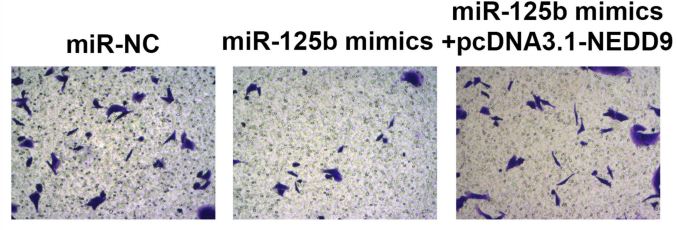

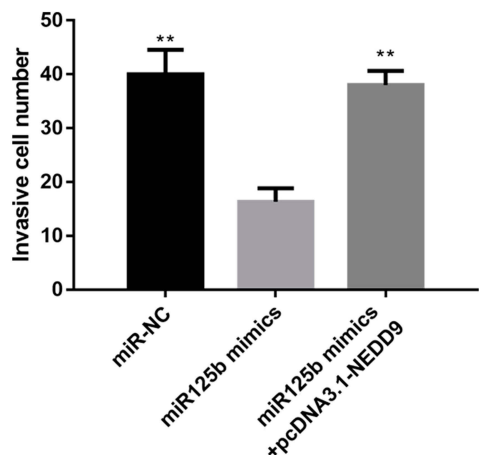

Figure 6 NEDD9 reverses the effect of miR-125b on proliferation and invasion of CAPANI cells.

Notes: (A) Relative mRNA expression of NEDD9 in CAPANI cells with different transfections; (B) Western blot analysis and band intensity analysis of NEDD9 in CAPANI cells with different transfections; (C) proliferation of CAPANI cells determined by MTT after different transfections; (D) BrdU incorporation assay for cell proliferation; (E) representative images of cell invasion across the membrane. Data presented as mean $\pm S D(n=3)$, **p<0.0I vs the miR-I25b mimics group.

miR-125b overexpression, nuclear DNA replication activity was significantly reduced, whereas knockdown of miR$125 \mathrm{~b}$ levels led to a significant increase in nuclear DNA replication activity. Flow cytometry revealed that overexpressed miR-125b had an anti-apoptotic effect on pancreatic cancer cells, whereas the inhibition of miR-125b expression promoted the pro-apoptotic ability of CAPAN1 cells. A series of biochemical switches trigger cell cycle progression, and there are three major cell cycle regulatory checkpoint transitions throughout the cell cycle events: ${ }^{20-22}$ The starting points are the G1/S checkpoint, and the G2/M checkpoint. Cell cycle experiments showed that increasing the expression of miR-125b decreased the number of cells in the S and G2 phases, and increased the percentage of cells in the G0/G1 phase. Moreover, when the expression level of miR-125b increased, the cell invasive ability is correspondingly lowered. In addition, the recovery of miR-125b in CAPAN1 cells significantly regulated the apoptosis-related and PI3K/AKT signaling pathway proteins.

NEDD9, which is also a non-catalytic scaffold protein and a member of the Crk-related substrate (CAS) family, was originally identified by its expression pattern of developmental regulation in the brains of early embryonic mice. ${ }^{23,24}$ 

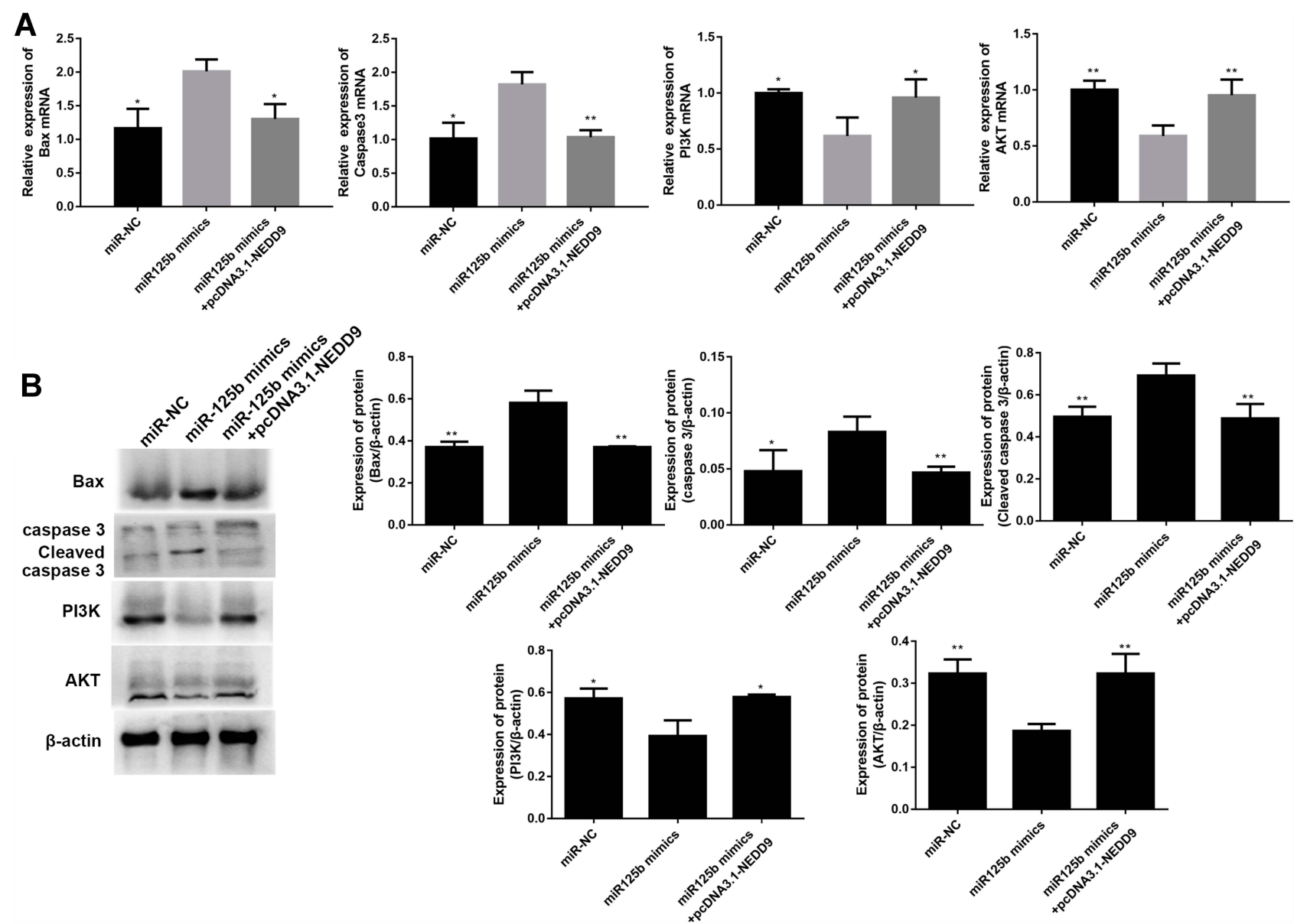

Figure 7 NEDD9 reverses the effect of miR-125b on apoptosis-related proteins and PI3K/AKT signaling pathways in CAPANI cells.

Notes: (A) The mRNA levels of BAX, caspase 3, PI3K and AKT; (B) the protein levels of BAX, caspase 3 , cleaved caspase 3 , PI3K and AKT. The mean values \pm SD ( $n=3$ ) are shown. ${ }^{*} p<0.05,{ }^{* *} p<0.01$ vs the miR-125b mimic group.

NEDD9 has been implicated in the invasion ability of several tumors and has been speculated to be a candidate biomarker of invasiveness for lung cancer, breast cancer, melanoma, colon cancer and pancreatic cancer. ${ }^{17,25,26}$ The function of NEDD9 related to cancer metastasis has focused on the FAK, Src and Akt signaling pathways. ${ }^{27}$ The interactions of NEDD9 with FAK and Src led to the tyrosine phosphorylation of NEDD9 to create binding sites for effector proteins to facilitate cell migration. ${ }^{28}$ NEDD9 also promoted pancreatic cancer progression via interacting with miR-145 or activating Akt and ERK signaling. ${ }^{25,29}$ The current study demonstrated that miR-125b levels were down-regulated in human pancreatic cancer tissues, whereas NEDD9 expression was upregulated. When miR-125b was overexpressed, the NEDD9 levels in CAPAN1 cells decreased. The simultaneous effect on cell proliferation and invasion suggested that miR-125b may be associated with the control of NEDD9 in PDAC. Also, the levels of NEDD9 and miR-125b are negatively correlated in pancreatic cancer. The luciferase activity assay $^{30,31}$ further supports this hypothesis, where the data proves that miR-125b was capable of directly targeting predicted region of NEDD9. NEDD9 was also shown to impair the function of miR-125b.

The results of our study indicate that further studies are crucial for understanding and describing the various roles of miR-125b in the aggressiveness of pancreatic cancer cells or patients or the tumor-suppression of miR-125b in vivo.

Taken together, these data demonstrated that miR-125b played a role in PDAC by targeting NEDD9.

\section{Conclusion}

Our research demonstrated that miR-125b was downregulated in PDAC, and that miR-125b recovery inhibited PDAC cell proliferation, invasion and apoptosis by directly binding post-transcriptional levels to negatively regulate NEDD9. 


\section{Acknowledgment}

This study was supported by Key Talents Project of "Strengthening Health through Science and Education" of Wuxi Health and Family Planning Commission (No. ZDRC039), Top Talents Project of "Six-one Project" for High-level Health Talents in Jiangsu Province (No. LGY2018016) and Appropriate Technology Promotion Project of Wuxi Health and Family Planning Commission (No. T201804).

\section{Disclosure}

The authors report no conflicts of interest in this work.

\section{References}

1. Longnecker DS, Morgan RG. Diet and Cancer of the Pancreas: Epidemiological and Experimental Evidence. In: Reddy BS, editor Diet, Nutrition and Cancer: A Critical Evaluation. London: CRC Press; 2018:11-26.

2. Raphael BJ, Hruban RH, Aguirre AJ, et al. Integrated genomic characterization of pancreatic ductal adenocarcinoma. Cancer Cell. 2017;32:185-203. doi:10.1016/j.ccell.2017.07.007

3. Chen W, Zheng R, Zhang S, et al. Report of incidence and mortality in China cancer registries, 2009. Chin J Cancer Res. 2013;25:10.

4. Torre LA, Siegel RL, Jemal A. Lung Cancer Statistics. Lung Cancer and Personalized Medicine. Springer; 2016:1-19.

5. Lin Q-J, Yang F, Jin C, Fu D-L. Current status and progress of pancreatic cancer in China. World J Gastroenterol. 2015;21:7988.

6. Muckenhuber A, Berger AK, Schlitter AM, et al. Pancreatic ductal adenocarcinoma subtyping using the biomarkers hepatocyte nuclear factor-1A and cytokeratin-81 correlates with outcome and treatment response. Clin Cancer Res. 2018;24:351-359. doi:10.1158/10780432.CCR-17-2180

7. Qu S, Yang X, Song W, et al. Downregulation of lncRNA-ATB correlates with clinical progression and unfavorable prognosis in pancreatic cancer. Tumour Biol. 2016;37:3933-3938. doi:10.1007/ s13277-015-4252-y

8. Amirkhah R, Schmitz U, Linnebacher M, Wolkenhauer O, Farazmand A. MicroRNA-mRNA interactions in colorectal cancer and their role in tumor progression. Genes Chromosomes Cancer. 2015;54:129-141.

9. Ye W, Lv Q, Wong C-KA, et al. The effect of central loops in miRNA: MRE duplexes on the efficiency of miRNA-mediated gene regulation. PloS One. 2008;3:e1719.

10. Schetter AJ, Leung SY, Sohn JJ, et al. MicroRNA expression profiles associated with prognosis and therapeutic outcome in colon adenocarcinoma. JAMA. 2008;299:425-436. doi:10.1001/ jama.299.4.425

11. Yu J, Li A, Hong S-M, Hruban RH, Goggins MJ. MicroRNA alterations of pancreatic intraepithelial neoplasias. Clin Cancer Res. 2012;18:981-992.

12. Bao B, Ali S, Ahmad A, et al. Differentially expressed miRNAs in cancer-stem-like cells: markers for tumor cell aggressiveness of pancreatic cancer. Stem Cells Dev. 2014;23:1947-1958.

13. Jung DE, Wen J, Oh T, Song SY. Differentially expressed microRNAs in pancreatic cancer stem cells. Pancreas. 2011;40:1180-1187. doi:10.1097/MPA.0b013e318221b33e
14. Ottaviani S, Castellano LJM. oncology c. microRNAs: novel regulators of the TGF- $\beta$ pathway in pancreatic ductal adenocarcinoma. Mol cell oncol. 2018;5:e1499066.

15. Shagisultanova E, Gaponova AV, Gabbasov R, Nicolas E, Golemis EA. Preclinical and clinical studies of the NEDD9 scaffold protein in cancer and other diseases. Gene. 2015;567:1-11. doi:10.1016/j.gene.2015.04.086

16. Dai J, Van Wie PG, Fai LY, et al. Downregulation of NEDD9 by apigenin suppresses migration, invasion, and metastasis of colorectal cancer cells. Toxicol Appl Pharmacol. 2016;311:106-112. doi:10.1016/j.taap.2016.09.016

17. Yu W, Ding J, He M, et al. Estrogen receptor $\beta$ promotes the vasculogenic mimicry (VM) and cell invasion via altering the lncRNA-MALAT1/miR-145-5p/NEDD9 signals in lung cancer. Oncogene. 2019;38:1225-1238. doi:10.1038/s41388-018-0463-1

18. Ferlay J. Cancer incidence, mortality and prevalence worldwide. GLOBOCAN2002. 2004.

19. Zhang Y, Lieberman J. Abstract B057: enhancing immunotherapy for triple-negative and HER2+ breast cancer using EpCAM aptamer-siRNA mediated gene knockdown. AACR. 2019:B057.

20. Gordon A, Greenfield EM, Eastell R, Kiss-Toth E, Wilkinson JM. Individual susceptibility to periprosthetic osteolysis is associated with altered patterns of innate immune gene expression in response to proinflammatory stimuli. J Orthop Res. 2010;28:1127-1135. doi:10. 1002/jor.21135

21. Wei T, Wang M, Wang M, Gan L-Y, Li XJ. Relationship of sRANKL level and vascular calcification score to cardiovascular events in maintenance hemodialysis patients. Blood Purif. 2009;28:342-345.

22. Daniel C, Duffield J, Brunner T, et al. Matrix metalloproteinase inhibitors cause cell cycle arrest and apoptosis in glomerular mesangial cells. J Pharmacol Exp Ther. 2001;297:57-68.

23. Shi R, Wang L, Wang T, Xu J, Wang F, Xu M. NEDD9 overexpression correlates with the progression and prognosis in gastric carcinoma. Med Oncol. 2014;31:852.

24. Deneka AY, Nikonova A, Kopp M, et al. Nedd9 controls glycolysis and autophagy in non-small cell lung cancer (NSCLC). AACR. 2019.

25. Han T, Yi XP, Liu B, Ke MJ, Li YX. MicroRNA-145 suppresses cell proliferation, invasion and migration in pancreatic cancer cells by targeting NEDD9. Mol Med Report. 2015;11:4115-4120. doi: $10.3892 / \mathrm{mmr} .2015 .3294$

26. Gu Y, Lu J, Chen C, Zheng F. NEDD9 overexpression predicts poor prognosis in solid cancers: a meta-analysis. Onco Targets Ther. 2019;12:4213-4222. doi:10.2147/OTT.S205760

27. Wang Z, Shen M, Lu P, Li X, Zhu S, Yue S. NEDD9 may regulate hepatocellular carcinoma cell metastasis by promoting epithelial-mesenchymal-transition and stemness via repressing Smad7. Oncotarget. 2017;8:1714-1724. doi:10.18632/oncotarget.13 852

28. Oneill GM, Seo S, Serebriiskii IG, Lessin SR, Golemis EA. A new central scaffold for metastasis: parsing HEF1/Cas-L/NEDD9. Cancer Res. 2007;67:8975-8979. doi:10.1158/0008-5472.CAN-07-1328

29. Zhou R, He M, Yu Z, et al. Baicalein inhibits pancreatic cancer cell proliferation and invasion via suppression of NEDD9 expression and its downstream Akt and ERK signaling pathways. Oncotarget. 2017;8:56351-56363. doi:10.18632/oncotarget. 16912

30. Brennan JC, Tillitt DE. Development of a dual luciferase activity and fluorescamine protein assay adapted to a 384 micro-well plate format: reducing variability in human luciferase transactivation cell lines aimed at endocrine active substances. Toxicol In Vitro. 2018;47:18-25. doi:10.1016/j.tiv.2017.10.030

31. Remy I, Michnick SW. A highly sensitive protein-protein interaction assay based on Gaussia luciferase. Nat Methods. 2006;3:977. 


\section{Publish your work in this journal}

Cancer Management and Research is an international, peer-reviewed open access journal focusing on cancer research and the optimal use of preventative and integrated treatment interventions to achieve improved outcomes, enhanced survival and quality of life for the cancer patient.
The manuscript management system is completely online and includes a very quick and fair peer-review system, which is all easy to use. Visit http://www.dovepress.com/testimonials.php to read real quotes from published authors.

Submit your manuscript here: https://www.dovepress.com/cancer-management-and-research-journal 